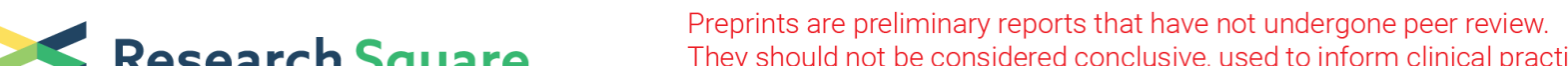 $\begin{array}{ll}\text { Research Square } & \text { They should not be considered conclusive, used to inform clinical practice, } \\ \text { or referenced by the media as validated information. }\end{array}$
}

\section{Downregulation of KIF21B by miR-132-3p suppresses cellular functions in gastric cancer via regulating Wnt/ $\beta$-catenin signaling pathway}

Bingtian Liu

Shandong University Cheeloo College of Medicine

Ling Qiang

Shandong Cancer Hospital: Shandong Cancer Hospital and Institute

Bingxin Guan

Shandong University Cheeloo College of Medicine

Zhipeng Ji ( $\nabla$ jizhi_peng0304@126.com )

Shandong University Cheeloo College of Medicine https://orcid.org/0000-0002-6052-0679

\section{Research Article}

Keywords: Gastric cancer, KIF21B, miR-132-3p, Wnt/ $\beta$-catenin signaling

Posted Date: September 2nd, 2021

DOI: https://doi.org/10.21203/rs.3.rs-850811/v1

License: (a) (i) This work is licensed under a Creative Commons Attribution 4.0 International License.

Read Full License 


\section{Abstract}

Recently, kinesin family member 21B (KIF21B) has been reported to be an oncogene in non-small cell lung cancer and hepatocellular carcinoma. However, the functional role and related mechanisms underlying gastric cancer (GC) pathogenesis remain largely uncovered. Here, we first found that the expression of KIF21B was upregulated in GC tissues compared with adjunct normal tissues by analysis of Oncomine microarray gene expression datasets and clinical specimens. Knockdown of KIF21B significantly suppressed the proliferation, migration and invasion in GC cell lines using CCK-8 assay and transwell assay. By luciferase reporter assay, KIF21B was confirmed as the target of miR-132-3p in GC cells and suppressed after miR-132-3p overexpression. Moreover, miR-132-3p was down-regulated and inversely correlated with KIF21B expression in GC tissues. Further functional experiments demonstrated that overexpression of KIF21B remarkedly reversed the suppressive effects of miR-132-3p overexpression on GC cell proliferation, migration and invasion. Furthermore, western blot analysis manifested that miR132-3p overexpression downregulated the protein levels of Wnt1, c-Myc, $\beta$-catenin, PCNA and N-cadherin, and upregulated E-cadherin expression in GC cells, which were all alleviated after KIF21B overexpression. In summary, our findings provide the first evidence that down-regulation of KIF21B by miR-132-3p suppresses cellular functions in gastric cancer via regulating $\mathrm{Wnt} / \beta$-catenin signaling.

\section{Introduction}

Gastric cancer (GC) is considered as the fifth most frequently occurred malignancy and third leading cause of tumor-associated death worldwide, remaining a global health problem [1,2]. Despite advances in comprehensive therapies, including surgery, adjuvant chemotherapy and radiation interventions, the prognosis of GC patients remains poor primarily ascribed to the diagnosis of GC at an advanced stage and uncontrolled metastasis [3, 4]. Therefore, having a better understanding the molecular mechanisms associated with GC pathogenesis will be great importance to improve the clinical outcomes of GC patients.

Kinesin superfamily proteins (KIFs), as microtubule-dependent molecular motors, perform a series of cellular processes, including mitosis, motility and organelle transportation [5-7]. KIFs have been recently found to be aberrantly expressed and participated in tumorigenesis by modulating chromosomal and spindle movement [8-10]. As a member of the KIFs, kinesin family member 21B (KIF21B), located at $1 \mathrm{q} 32.1$, contains a motor domain, a stalk and a tail domain binding to microtubules, which is essential for neuronal morphology [11]. Interestingly, accumulating evidence has demonstrated that KIF21B was involved in several diseases, especially tumorigenesis. For instance, abundant KIF21B expression is associated with severe multiple sclerosis and Alzheimer's disease pathology with accelerated neurodegeneration $[12,13]$. The influence of KIF21B may be relevant to mechanism underlying autism spectrum disorders etiology [14]. Zhao et al [15] showed that KIF21B was identified as an independent risk factor for overall survival and disease-free survival in patients with hepatocellular carcinoma. Sun et al [16] not only manifested that high expression of KIF21B predicted a poor prognosis of patients with non-small cell lung cancer (NSCLC), but also promoted the proliferation, migration and invasion of A549 
and $\mathrm{H} 1299$ cells. However, the expression pattern and functional role of KIF21B in GC have not yet been investigated until now.

MicroRNAs (miRNAs/miRs), a type of small non-coding RNAs, have been frequently demonstrated to function as pivotal regulators in tumorigenesis though modulating various biological processes, including proliferation, invasion and differentiation via binding to the $3^{\prime}$ untranslated region (3'-UTR) of target genes [17-20]. In recent years, miR-132-3p has been reported to be aberrantly expressed in tumor tissues and serve an important regulator in tumor cell functions. For example, miR-132-3p suppressed the migration and invasion of breast carcinoma cells through lysosomal-associated protein transmembrane 4 beta (LAPTM4B) by mediating epithelial-mesenchymal transition signals [21]. Ectopic miR-132-3p aggravated cell apoptosis and inhibited cell proliferation in mantle cell lymphoma [22]. In addition, miR132-3p functions as a tumor suppressor in colorectal cancer $[23,24]$, osteosarcoma $[25,26]$ and bladder carcinoma [27]. Here, our previous investigation revealed that KIF21B was a potential target gene of miR132-3p. Moreover, Zhang et al [28] and Wang et al [29] consistently demonstrated that miR-132-3p expression was significantly altered between tumor tissues and normal tissues derived from patients with GC by microRNA profiling. Based on these facts, we speculated that miR-132-3p/KIF21B axis plays a critical role in regulating cell functions in GC cells.

To validate our hypothesis, we first determined the expression pattern of KIF21B in GC tissues by searching Oncomine database and collecting clinical specimens. Then, we performed loss-of-function assays to analyze the functional role of KIF21B in GC cells. Furthermore, we further explored whether $\mathrm{KIF} 21 \mathrm{~B}$ was the downstream regulator involved in miR-132-3p regulating GC cell functions by modulating Wnt/ $\beta$-catenin signaling. Our findings may provide novel insights into the treatment of GC.

\section{Materials And Methods}

\section{Oncomine gene expression analysis}

We first examined the expression of KIF21B gene in GC by searching microarray gene expression datasets derived from Oncomine database (www.oncomine.com). In brief, the Cancer Type was defined as Gastric Cancer, Data Type was mRNA, and Analysis Type was Cancer versus Normal Analysis. Total three datasets, including Wang Gastric [30], Chen Gastric [31] and DErrico Gastric [32] were included in our analysis. The log-transformed, median-centered and normalized expression values of KIF21B were extracted, analyzed and read from the scatterplot accordingly.

\section{Tissue specimens}

Total thirty pairs of fresh tumor tissues and matched adjacent normal stomach mucosa tissues were collected from GC patients who received radical gastrectomy at the Second Hospital, Cheeloo College of Medicine, Shandong University (Shandong Province, China). All patients did not receive radiotherapy and chemotherapy before surgery. In addition, paraffin-embedded specimens of GC were also obtained to evaluate the KIF21B protein expression. Informed consent was obtained from all patients. This study was 
conducted in accordance with Helsinki Declaration and approved by the Medical Ethics Committee of the Second Hospital, Cheeloo College of Medicine, Shandong University (Shandong Province, China).

\section{Immunohistochemistry}

Immunohistochemistry staining was performed to assess the protein expression of KIF21B in tissue samples using the EliVisionTMplus kit (Maixin, Fuzhou, China) according to the instructions provided. Briefly, paraffin-embedded tissues were sliced into $5-\mu \mathrm{m}$ thick sections. The tissue sections were deparaffinized in xylene and rehydrated in gradient ethanol. After subjected to antigen retrieval by heated citrate buffer, sections were blocked in $3 \%(\mathrm{v} / \mathrm{v})$ hydrogen peroxide for $30 \mathrm{~min}$ and incubated overnight at $4{ }^{\circ} \mathrm{C}$ with anti-KIF21B antibody (ab121931; Abcam, Cambridge, UK). Afterwards, the sections were washed with twice with Tris-buffered saline and then incubated with horseradish peroxidase-conjugated secondary antibody for $2 \mathrm{~h}$ at room temperature. The immunohistochemistry staining results were evaluated by two independent pathologists according to the multiplication of staining proportion score (0-4: $0,0-5 \% ; 1,6-20 \% ; 2,21-60 \% ; 3,61-75 \%$; or $4,76-100 \%)$ and staining intensity score $(0$, no staining; 1 , weak; 2 , moderate; 3 , strong staining) as the final score of KIF21B protein expression. Tissue sections with immunoreactivity score scaling $0-2,2-4$ and over 4 were considered to be weak staining (-+), moderate staining $(+)$ and strong staining $(++)$, respectively.

\section{Cell culture and transfection}

Two GC cell lines, including AGS and SNU-5 were purchased from the Cell Bank of China Academy of Sciences (Shanghai, China) and cultured RPMI-1640 medium (Thermo Fisher Scientific, Inc., Waltham, MA, USA) supplemented with $10 \%$ fetal bovine serum (FBS, Gibco) at $37^{\circ} \mathrm{C}$ in the atmosphere containing $5 \% \mathrm{CO}_{2}$. The sequences of small interfering RNA targeting KIF21B (si-KIF21B\#1 and si-KIF21B\#2), si-NC, miR-132-3p mimics and miR-NC were synthesized by GenePharma Co., Ltd. (Shanghai, China). The pcDNA3.1 containing the open reading frame of KIF21B or empty vector were purchased from GenScript (Nanjing, China). After incubating GC cells in 12-well plates at a density of $8 \times 10^{4}$ cells per well, the cells were transfected with the above oligonucleotides for $48 \mathrm{~h}$ using Lipofectamine 2000 (Thermo Fisher Scientific, Inc.).

\section{Reverse transcription quantitative PCR analysis}

Total RNA sample was extracted from tissue specimens or cell lines using TRIzol reagent (Invitrogen). Reverse transcription was conducted by One Step PrimeScript miRNA complementary DNA Synthesis Kit (Takara, Dalian, China) for miR-132-3p and a HiFiScript cDNA Synthesis Kit (CWBIO, Beijing, China) for KIF21B. Next, reverse transcription quantitative PCR was performed to determine the expression of miR132-3p and KIF21B using SYBR Green Human miRNA Assay Kit and a SYBR Premix Ex Taq II kit (Takara, Japan), respectively. The primer sequences were used as follows: miR-132-3p (forward: 5囚-

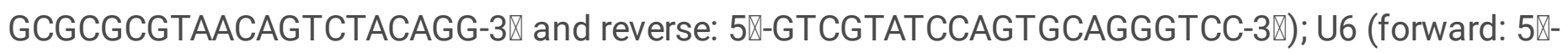
CTCGCTTCGGCAGCACATATACT-3囚 and reverse: 5囚-CGCTTCACGAATTTGCGTGT-3囚); KIF21B (forward: 5'CGA GGAGACGGATGAGAACG-3' and reverse, 5'-CCACCAGGCTCTCTTCACTG-3'); $\beta$-actin (forward: 5'- 
CCCGAGCCGTGTTTCCT-3' and reverse: 5'-GTCCCAGTTGGT GACGATGC-3'). Relative miR-132-3p and KIF21B mRNA expression were normalized against the endogenous control U6 and $\beta$-actin, respectively using the $2-\Delta \Delta$ Ct method [33].

\section{Cell counting kit-8 (CCK-8) assay}

Transfected cells at a density of $4 \times 10^{3}$ cells per well were seeded into 96-well plates and cultured at 37 ${ }^{\circ} \mathrm{C}$. At $0,24,48$ and $72 \mathrm{~h}$, respectively, cells in each well were incubated with $10 \mu \mathrm{l}$ of CCK-8 solution (Solarbio Science \& Technology, Beijing, China) for another $2 \mathrm{~h}$ at $37^{\circ} \mathrm{C}$. Afterwards, the optical density (OD) value at $450 \mathrm{~nm}$ was measured with a microplate reader.

\section{Transwell migration and invasion assay}

For migration assay, approximately $4 \times 10^{4}$ transfected cells in $200 \mu \mathrm{l}$ of serum-free medium were plated into the upper chamber (24-well insert; 8- $\mu$ m pore size; Corning Costar, Corning, NY, USA), while $600 \mu \mathrm{l}$ complete medium (with 10\% FBS) as chemoattractant was added into the lower chamber. After $24 \mathrm{~h}$ incubation, the cells that migrated to the lower chamber were fixed with $4 \%$ paraformaldehyde for $30 \mathrm{~min}$ and stained with $0.1 \%$ crystal violet for $10 \mathrm{~min}$. The number of migrated cells was quantified under a microscope (magnification, $\times 100$ ) in five random microscope fields. The procedure of invasion assay was similar to the migration assay except for the addition of $50 \mu$ l Matrigel on the membranes of transwell inserts and incubation time of $48 \mathrm{~h}$.

\section{Bioinformatic analysis and luciferase reporter assay}

The potential miRNAs that target KIF21B were predicted by TargetScan (www.targetscan.org/vert_71/). From all these predictions, we selected miR-132-3p for further analysis. The mRNA 3'-UTR of KIF21B, carrying the predicted binding site or mutant binding site of miR-132-3p, was amplified by PCR and cloned into pmirGLO (Promega, Madison, Wisconsin), which were named as KIF21B wild-type (WT) and mutant (MUT) constructs, respectively. Next, cells were co-transfected with KIF21B WT or KIF21B MUT and miR-132-3p mimics or miR-NC using the transfection reagent Lipofectamine 2000. After $48 \mathrm{~h}$ incubation, cells were harvested and relative luciferase activity was measured via a Dual-luciferase reporter assay system (Promega).

\section{Western blot analysis}

Total protein sample was extracted with RIPA lysis buffer with protease inhibitor (Solarbio Science \& Technology Co., Ltd., Beijing, China) and protein concentration was determined by a BCA kit (Beyotime, Shanghai, China). Then, equal amount of protein sample was separated by $10 \%$ SDS-PAGE and transferred onto PVDF membranes (Millipore, Billerica, MA, USA). After blocked with $5 \%$ non-fat milk, the membranes were incubated with primary antibodies against KIF21B, Wnt1, c-Myc, $\beta$-catenin, PCNA, Ecadherin, N-cadherin and GAPDH (All from Abcam, Cambridge, UK) at $4{ }^{\circ} \mathrm{C}$ overnight. Subsequently, the membranes were incubated with horseradish peroxidase-coupled secondary antibody for $2 \mathrm{~h}$ at room 
temperature, followed by detection of protein signals with an enhanced chemiluminescence reagent (EMD Millipore, Billerica, MA, USA).

\section{Statistical analysis}

All quantitative data were presented as mean \pm SD from three independent experiments. All statistical analyses were performed using GraphPad Prism 6.0 (GraphPad Software, Inc., San Diego, California, USA). Differences between two groups were assessed by paired Student's t-test. Differences among three groups were evaluated by one-way analysis of variance (ANOVA), followed by Dunnett's test or Tukey's test. The correlation between miR-132-3p and KIF21B was evaluated using Pearson's correlation coefficient analysis. The $p$-value less than 0.05 was considered to be statistically significant.

\section{Results}

\section{The expression of KIF21B was upregulated in GC tissues}

To investigate the expression pattern of KIF21B in GC, we determined the differential mRNA expression of KIF21B between GC tissues and normal gastric tissues by analysis of the Oncomine microarray gene expression datasets. As shown in Fig. 1A, the expression of KIF21B was significantly increased in gastric cancer tissues $(p=7.73 \mathrm{E}-4)$ compared with the corresponding normal tissues in the Wang Gastric dataset, in diffuse gastric adenocarcinoma $(p=0.002)$ and gastric intestinal type adenocarcinoma $(p=$ $0.005)$ compared with normal tissues in Chen Gastric dataset, as well as in gastric intestinal type adenocarcinoma $(p=0.010)$ and gastric mixed adenocarcinoma $(p=0.016)$ compared with normal tissues in DErrico Gastric dataset. To confirm the upregulation of KIF21B in GC, reverse transcription quantitative PCR was performed to analyze the expression of KIF21B mRNA in thirty pairs of fresh GC tissue and matched adjacent normal tissues. The results showed that the expression of KIF21B mRNA was significantly upregulated in GC tissues compared with adjacent tissues (Fig. 1B). Moreover, the results from IHC analysis (Fig. 1C) indicated that representative photomicrographs of different degrees of KIF21B expression intensity were observed in the cytoplasm. Expression of the KIF21B protein was detected in $78.6 \%$ of GC-derived tissues and in $18.5 \%$ of noncancerous tissues, which suggested that KIF21B was highly expressed in GC tissues.

\section{Knockdown of KIF21B inhibited the proliferation, migration and invasion of GC cells}

Next, we explored the functional role of KIF21B in GC cells by performing loss-of-function assays in AGS and SNU-5 cells. At first, the expression of KIF21B protein was suppressed by transfecting with two different siRNAs in AGS and SNU-5 cells, as demonstrated by western blot analysis (Fig. 2A). CCK-8 assay showed that either si-KIF21B\#1 or si-KIF21B\#2 transfection significantly suppressed cell proliferation ability in AGS and SNU-5 cells (Fig. 2A). Notably, si-KIF21B\#2 generated more powerful suppressive effects on KIF21B expression and cell proliferation ability, in comparison with si-KIF21B\#1, which was thus selected for the subsequent in vitro experiments. Subsequently, transwell assay was applied to analyze the effects of KIF21B knockdown on GC cell migration and invasion. Our data showed 
that the number of migratory cells was remarkedly decreased in si-KIF21B\#2 group compared with si-NC group in both AGS and SNU-5 cells (Fig. 2C). Similarly, knockdown of KIF21B by si-KIF21B\#2 transfection significantly reduced the number of invasive cells in AGS and SNU-5 cells (Fig. 2D).

\section{Kif21b Was A Direct Target Of Mir-132-3p}

The miRNAs that target the expression of KIF21B were predicted using TargetScan 7.1 database, which led to the identification of miR-132-3p as a direct target gene. As depicted in Fig. 3A, miR-132-3p exhibited identical seed regions, with complementary binding to the 3'-UTR of KIF21B. Then, luciferase reporter assay was conducted to examine whether miR-132-3p directly targets KIF21B. The results showed that miR-132-3p mimics significantly suppressed the luciferase activity of AGS (Fig. 3B) and SNU-5 (Fig. 3C) cells transfected with KIF21B WT plasmid. However, the luciferase activity remained unchanged when the cells were transfected with KIF21B MUT without the miR-132-3p binding sites. What's more, overexpression of miR-132-3p significantly downregulated the expression of KIF21B mRNA (Fig. 3D) and protein (Fig. 3E) levels in both AGS and SNU-5 cells. These findings suggested that miR-132-3p could negatively regulate KIF21B expression through its 3'-UTR. In addition, we analyzed the expression of miR132-3p in GC tissues. As shown in Fig. 3F, the expression of miR-132-3p was significantly down-regulated in GC tissues compared with adjacent tissues. Furthermore, the expression of miR-132-3p was inversely correlated with KIF21B expression in 30 cases of GC tissues (Fig. 3G, $r=-0.4132, p=0.0232$ ).

\section{Overexpression of KIF21B reversed the suppressive effects of miR-132-3p on GC cell proliferation, migration and invasion}

Since miR-132-3p was identified to target KIF21B and negative correlated with KIF21B expression in GC tissues, we thus explored whether miR-132-3p regulated GC cell functions by targeting KIF21B. At first, the expression of miR-132-3p was overexpressed in AGS and SNU-5 cells after transfection with miR-132-3p mimics, as demonstrated by reverse transcription quantitative PCR (Fig. 4A). Then, AGS and SNU-5 cells were transfected with miR-NC, miR-132-3p mimics or miR-132-3p mimics + KIF21B, respectively. As shown in Fig. 4B, downregulated KIF21B protein level induced by miR-132-3p overexpression was attenuated after KIF21B overexpression plasmid transfection in both AGS and SNU-5 cells. The in vitro experiments indicated that ectopic KIF21B expression effectively reversed the effects of miR-132-3p overexpression on cell proliferation (Fig. 4C), migration (Fig. 4D) and invasion (Fig. 4E) abilities in AGS and SNU-5 cells.

\section{MiR-132-3p suppressed the Wnt/ $\beta$-catenin signaling pathway in GC cells via repressing KIF21B}

It has been reported that $\mathrm{Wnt} / \beta$-catenin signaling pathway participates in regulating tumor cell proliferation, migration and invasion. Thus, we next investigated whether $\mathrm{Wnt} / \beta$-catenin signaling pathway was involved in the regulation of GC cells induced by miR-132-3p targeting KIF21B. As shown in Fig. 5A, miR-132-3p overexpression obviously suppressed the expression of Wnt1, c-Myc and $\beta$-catenin, which was reversed after KIF21B overexpression in AGS cells. In addition, we found that miR-132-3p 
overexpression downregulated the expression of PCNA and N-cadherin, and upregulated E-cadherin expression in AGS cells, which was reversed after co-transfection with miR-132-3p mimics and KIF21B. Similarly, we observed that KIF21B overexpression abolished the effects of miR-132-3p overexpression on the protein levels of Wnt1, C-Myc, $\beta$-catenin, PCNA, E-cadherin and N-cadherin in SNU-5 cells (Fig. 5B). These results indicated that KIF21B was a direct target of miR-132-3p involved in Wnt/ $\beta$-catenin pathway in GC cells.

\section{Discussion}

In the current study, we first observed that KIF21B expression was upregulated in GC tissues compared with adjacent normal tissues by analysis of the Oncomine microarray gene expression datasets and clinical specimens. Next, we performed loss-of-function experiments to demonstrate that silencing of KIF21B suppressed the proliferation, migration and invasion of AGS and SNU-5 cells. Actually, KIFs play crucial roles in mitotic cell division, which make them involved in tumorigenesis [34, 35]. Similarly, upregulation of KIF14 could promote cancer metastasis in gastric cancer [36] and promote cell proliferation in colorectal cancer [37]. KIF11 inhibition significantly reduced cell viability and colony formation, inhibited migration and invasion in breast cancer [38]. Knockdown of KIF23 resulted in a marked inhibition of cell proliferation of GC in mice, with significant downregulation of Ki67 and PCNA expression [39]. Consistent with our data, KIF21B was upregulated in hepatocellular carcinoma and NSCLC tissues, which promoted the corresponding tumor cell proliferation, migration and invasion [15, 16]. Herein, our data highlight the oncogenic role of KIF21B in GC.

By bioinformatic analysis and luciferase reporter assay, we further demonstrated that KIF21B was a potential target of miR-132-3p in AGS and SNU-5 cells. What's more, decreased miR-132-3p expression was found to be inversely correlated with KIF21B mRNA levels in 30 cases of GC tissues. The rescue experiments manifested that overexpression of KIF21B significantly alleviated the suppressive effects of miR-132-3p on cell proliferation, migration and invasion in AGS and SNU-5 cells. These data suggested that miR-132-3p inhibited the cellular functions by directly targeting KIF21B in GC cells. In fact, miR-132$3 p$ expression was demonstrated to be more frequently downregulated in human cancer, including colorectal cancer [40], pancreatic carcinoma [41] and glioma [42, 43]. Functionally, the suppressive effects of miR-132-3p have been reported on cell proliferation, migration and invasion in breast cancer [21], mantle cell lymphoma [22], colorectal cancer [23, 24], osteosarcoma [25, 26] and bladder carcinoma [27]. Notably, He et al showed that inhibition of miR-132-3p enhanced GC cell proliferation and migration by targeting mucin 13 (MUC13) [44]. Despite the suppressive role of miR-132-3p has been reported already, there are some major differences between it and our study as follows: 1) We performed gain-offunction assay to investigate the functional role of miR-132-3p, rather than loss-of-function assay in the above study from He et al. 2) Different target genes of miR-132-3p were identified. 3) Different molecular mechanisms underlying miR-132-3p regulating GC cell functions. 4) In addition to migration and invasion, we analyzed cell proliferation here. Until now, several KIFs have been reported to be directly regulated by miRNAs involved in the development of tumors. For instance, miR-338-3p could directly bind to the 3 '-untranslated region (3'-UTR) of KIFC1, and ectopic miR-338-3p expression mimicked the 
inhibitory functions of KIFC1 silencing on renal cell carcinoma cells [45]. MiR-30a could specifically interact with KIF11 and suppress its expression in breast cancer [46]. Additionally, miR-206 directly suppresses KIF2A to inhibit ovarian cancer cell proliferation, migration, and invasion [47]. To our best knowledge, KIF21B has not been demonstrated to be a target of certain miRNA, which made the identification of KIF21B as the target of miR-132-3p provided a novel therapeutic target against the progression of GC.

It has been widely held that the Wnt/ $\beta$-catenin signaling pathway is frequently over-activated in multiple types of tumors, which plays a fundamental role in regulation of cellular proliferation and invasion [48]. What's more, Wnt/ $\beta$-catenin pathway is closely associated with EMT process and plays a critical role in metastasis [49]. Here, we found that miR-132-3p overexpression inhibited the activity of Wnt1, c-Myc and $\beta$-catenin, as key effector of Wnt/ $\beta$-catenin signaling pathway and inactivated EMT markers (E-cadherin and N-cadherin), which were all reversed after KIF21B overexpression in GC cells. Similar with our data, miR-132-3p suppressed the migration and invasion of tumor cells by regulating the EMT-correlated molecules in lung cancer [50,51] and cervical cancer [52]. It has been confirmed that KIF26B regulates cell invasion in breast cancer through driving epithelial-mesenchymal transition (EMT) [53]. KIF11 knockdown significantly upregulated E-cadherin expression, and downregulated the expression of $\mathrm{N}$ cadherin and Vimentin in breast cancer cells [38]. Although the association between miR-132-3p/KIF21B and $\mathrm{Wnt} / \beta$-catenin pathway has not been reported yet, we thus speculated miR-132-3p suppressed the migration and invasion of GC cells by targeting KIF21B-mediated Wnt/ $\beta$-catenin pathway based on the correlation of EMT process and Wnt/ $\beta$-catenin pathway.

\section{Conclusion}

In summary, we first demonstrated that KIF21B knockdown suppressed the proliferation, migration and invasion in GC cells. Moreover, we confirmed that KIF21B was the downstream regulator underlying miR132-3p exerted suppressive effects on GC cells by downregulating Wnt/ $\beta$-catenin pathway. Thus, we findings help us better understand targeting miR-132-3p/KIF21B axis as a promising therapeutic target for GC treatment.

\section{Abbreviations}

GC: gastric cancer; KIF21B, kinesin family member 21B; GC, gastric cancer; 3'-UTR, 3' untranslated region; LAPTM4B, lysosomal-associated protein transmembrane 4 beta; FBS, fetal bovine serum; CCK-8, Cell counting kit-8; OD, optical density; WT, wild-type; EMT, epithelial-mesenchymal transition

\section{Declarations}

\section{Ethics approval and consent to participate}


The present study was conducted in accordance with Helsinki Declaration and approved by the Ethics Committee of the Second Hospital, Cheeloo College of Medicine, Shandong University (Shandong Province, China). Written informed consent was obtained from all patients.

\section{Authors' contributions}

Supervision: Zhipeng Li; Interpretation of analysis of data: Bingtian Liu and Ling Qiang; Preparation of the manuscript: Bingxin Guan; Revision for important intellectual content: Ling Qiang. All authors read and approved the final manuscript.

\section{Availability of data and materials}

All results and data generated or analyzed during the present study are included in this published article or are available from the corresponding author on reasonable request.

\section{Acknowledgements}

Not applicable.

\section{Funding}

No funding was received.

\section{Consent for publication}

We have obtained consents to publish this paper from all the participants of this study.

\section{Competing interests}

The authors declare that they have no competing interests.

\section{References}

1. Bozkurt O, Firat ST, Dogan E, Cosar R, Inanc M, Ozkan M. The prognostic value of the change in neutrophil-to-lymphocyte ratio during first-line palliative chemotherapy in patients with metastatic gastric cancer: A retrospective study. J BUON. 2019;24:1992-9.

2. Sitarz R, Skierucha M, Mielko J, Offerhaus GJA, Maciejewski R, Polkowski WP. Gastric cancer: epidemiology, prevention, classification, and treatment. Cancer Manag Res. 2018;10:239-48.

3. Baba H, Kuwabara K, Ishiguro T, Kumamoto K, Kumagai Y, Ishibashi K, Haga N, Ishida H. Prognostic factors for stage IV gastric cancer. Int Surg. 2013;98:181-7.

4. Zhou Q, Lan X, Li N, Yuan D, Zhang J. Analysis of Prognostic Factors and Design of Prognosis Model for Patients with Stage IV Gastric Cancer Following First-Line Palliative Chemotherapy. Cancer Manag Res. 2020;12:10461-8. 
5. Hirokawa N, Tanaka Y. Kinesin superfamily proteins (KIFs): Various functions and their relevance for important phenomena in life and diseases. Exp Cell Res. 2015;334:16-25.

6. Hirokawa N, Noda Y. Intracellular transport and kinesin superfamily proteins, KIFs: structure, function, and dynamics. Physiol Rev. 2008;88:1089-118.

7. Hirokawa N, Noda Y, Tanaka Y, Niwa S. Kinesin superfamily motor proteins and intracellular transport. Nat Rev Mol Cell Biol. 2009;10:682-96.

8. Myers SM, Collins I. Recent findings and future directions for interpolar mitotic kinesin inhibitors in cancer therapy. Future Med Chem. 2016;8:463-89.

9. Liu X, Gong H, Huang K. Oncogenic role of kinesin proteins and targeting kinesin therapy. Cancer Sci. 2013;104:651-6.

10. Liu Y, Zhan P, Zhou Z, Xing Z, Zhu S, Ma C, Li Q, Zhu Q, Miao Y, Zhang J, Lv T, Song Y. The overexpression of KIFC1 was associated with the proliferation and prognosis of non-small cell lung cancer. J Thorac Dis. 2016;8:2911-23.

11. Muhia M, Thies E, Labonte D, Ghiretti AE, Gromova KV, Xompero F, Lappe-Siefke C, HermansBorgmeyer I, Kuhl D, Schweizer M, Ohana O, Schwarz JR, Holzbaur ELF, Kneussel M. The Kinesin KIF21B Regulates Microtubule Dynamics and Is Essential for Neuronal Morphology, Synapse Function, and Learning and Memory. Cell Rep. 2016;15:968-77.

12. Kreft $K L$, van Meurs $M$, Wierenga-Wolf AF, Melief MJ, van Strien ME, Hol EM, Oostra BA, Laman JD, Hintzen RQ. Abundant kif21b is associated with accelerated progression in neurodegenerative diseases. Acta Neuropathol Commun. 2014;2:144.

13. Hares K, Miners JS, Cook AJ, Rice C, Scolding N, Love S, Wilkins A. Overexpression of Kinesin Superfamily Motor Proteins in Alzheimer's Disease. J Alzheimers Dis. 2017;60:1511-24.

14. Gromova KV, Muhia M, Rothammer N, Gee CE, Thies E, Schaefer I, Kress S, Kilimann MW, Shevchuk O, Oertner TG, Kneussel M. Neurobeachin and the Kinesin KIF21B Are Critical for Endocytic Recycling of NMDA Receptors and Regulate Social Behavior. Cell Rep. 2018;23:2705-17.

15. Zhao HQ, Dong BL, Zhang M, Dong XH, He Y, Chen SY, Wu B, Yang XJ. Increased KIF21B expression is a potential prognostic biomarker in hepatocellular carcinoma. World J Gastrointest Oncol. 2020;12:276-88.

16. Sun ZG, Pan F, Shao JB, Yan QQ, Lu L, Zhang N. Kinesin superfamily protein 21B acts as an oncogene in non-small cell lung cancer. Cancer Cell Int. 2020;20:233.

17. Kunej T, Godnic I, Ferdin J, Horvat S, Dovc P, Calin GA. Epigenetic regulation of microRNAs in cancer: an integrated review of literature. Mutat Res. 2011;717:77-84.

18. Cortez MA, Anfossi S, Ramapriyan R, Menon H, Atalar SC, Aliru M, Welsh J, Calin GA. Role of miRNAs in immune responses and immunotherapy in cancer. Genes Chromosomes Cancer. 2019;58:244-53.

19. Jia XN, Yin SD, Wei Y, Chen L. MiR-182-5p inhibited proliferation and migration of ovarian cancer cells by targeting BNIP3. Eur Rev Med Pharmacol Sci. 2020;24:7912. 
20. Wang L, Wang Y, Du X, Yao Y, Wang L, Jia Y. MiR-216b suppresses cell proliferation, migration, invasion, and epithelial-mesenchymal transition by regulating FOXM1 expression in human nonsmall cell lung cancer. Onco Targets Ther. 2019;12:2999-3009.

21. Li S, Xu JJ, Zhang QY. MicroRNA-132-3p inhibits tumor malignant progression by regulating lysosomal-associated protein transmembrane 4 beta in breast cancer. Cancer Sci. 2019;110:3098109.

22. Wu B, Li J, Wang H, Wu Q, Liu H. MiR-132-3p serves as a tumor suppressor in mantle cell lymphoma via directly targeting SOX11. Naunyn Schmiedebergs Arch Pharmacol. 2020;393:2197-208.

23. He X, Ma J, Zhang M, Cui J, Yang H. Long Non-Coding RNA SNHG16 Activates USP22 Expression to Promote Colorectal Cancer Progression by Sponging miR-132-3p. Onco Targets Ther. 2020;13:428394.

24. Zhang M, Li Y, Wang H, Yu W, Lin S, Guo J. LncRNA SNHG5 affects cell proliferation, metastasis and migration of colorectal cancer through regulating miR-132-3p/CREB5. Cancer Biol Ther. 2019;20:524-36.

25. Guan H, Shang G, Cui Y, Liu J, Sun X, Cao W, Wang Y, Li Y. Long noncoding RNA APTR contributes to osteosarcoma progression through repression of miR-132-3p and upregulation of yes-associated protein 1. J Cell Physiol. 2019;234:8998-9007.

26. Li G, Liu K, Du X, Non-Coding L. RNA TUG1 Promotes Proliferation and Inhibits Apoptosis of Osteosarcoma Cells by Sponging miR-132-3p and Upregulating SOX4 Expression. Yonsei Med J. 2018;59:226-35.

27. Liu P, Li X, Guo X, Chen J, Li C, Chen M, Liu L, Zhang X, Zu X. Circular RNA DOCK1 promotes bladder carcinoma progression via modulating circDOCK1/hsa-miR-132-3p/Sox5 signalling pathway. Cell Prolif. 2019;52:e12614.

28. Zhang T, Liu C, Huang S, Ma Y, Fang J, Chen Y, A Downmodulated MicroRNA Profiling in Patients with Gastric Cancer, Gastroenterol Res Pract 2017; 2017: 1526981.

29. Wang J, Zhang H, Zhou X, Wang T, Zhang J, Zhu W, Zhu H, Cheng W. Five serum-based miRNAs were identified as potential diagnostic biomarkers in gastric cardia adenocarcinoma. Cancer Biomark. 2018;23:193-203.

30. Wang Q, Wen YG, Li DP, Xia J, Zhou CZ, Yan DW, Tang HM, Peng ZH. Upregulated INHBA expression is associated with poor survival in gastric cancer. Med Oncol. 2012;29:77-83.

31. Chen X, Leung SY, Yuen ST, Chu KM, Ji J, Li R, Chan AS, Law S, Troyanskaya OG, Wong J, So S, Botstein D, Brown PO. Variation in gene expression patterns in human gastric cancers. Mol Biol Cell. 2003;14:3208-15.

32. D'Errico M, de Rinaldis E, Blasi MF, Viti V, Falchetti M, Calcagnile A, Sera F, Saieva C, Ottini L, Palli D, Palombo F, Giuliani A, Dogliotti E. Genome-wide expression profile of sporadic gastric cancers with microsatellite instability. Eur J Cancer. 2009;45:461-9.

33. Livak KJ, Schmittgen TD, Analysis of Relative Gene Expression Data using Real-Time Quantitative PCR, Methods 2002; 25: 402-408. 
34. Huszar D, Theoclitou ME, Skolnik J, Herbst R. Kinesin motor proteins as targets for cancer therapy. Cancer Metastasis Reviews. 2009;28:197-208.

35. Lucanus AJ, Yip GW. Kinesin superfamily: roles in breast cancer, patient prognosis and therapeutics. Oncogene. 2018;37:833-8.

36. Yang Z, Li C, Yan C, Li J, Yan M, Liu B, Zhu Z, Wu Y, Gu Q. KIF14 promotes tumor progression and metastasis and is an independent predictor of poor prognosis in human gastric cancer. Biochim Biophys Acta Mol Basis Dis. 2019;1865:181-92.

37. Wang ZZ, Yang J, Jiang BH, Di JB, Gao P, Peng L, Su XQ. KIF14 promotes cell proliferation via activation of Akt and is directly targeted by miR-200c in colorectal cancer. Int $\mathrm{J}$ Oncol. 2018;53:1939-52.

38. Zhou J, Chen WR, Yang LC, Wang J, Sun JY, Zhang WW, He ZY, Wu SG. KIF11 Functions as an Oncogene and Is Associated with Poor Outcomes from Breast Cancer. Cancer Res Treat. 2019;51:1207-21.

39. Li XL, Ji YM, Song R, Li XN, Guo LS, KIF23 Promotes Gastric Cancer by Stimulating Cell Proliferation, Dis Markers 2019; 2019: 9751923.

40. Kara M, Yumrutas O, Ozcan O, Celik OI, Bozgeyik E, Bozgeyik I, Tasdemir S. Differential expressions of cancer-associated genes and their regulatory miRNAs in colorectal carcinoma. Gene. 2015;567:81-6.

41. Zhang H, Liu A, Feng X, Tian L, Bo W, Wang H, Hu Y. MiR-132 promotes the proliferation, invasion and migration of human pancreatic carcinoma by inhibition of the tumor suppressor gene PTEN. Prog Biophys Mol Biol. 2019;148:65-72.

42. Hangzhou W, Li X-T, Wu C. Zhi-Wu, Yan-Yan, Tian-Quan and Yang, miR-132 can inhibit glioma cells invasion and migration by target MMP16 in vitro, OncoTargets and therapy 2015.

43. Zhou K, Zhang C, Yao H, Zhang X, Zhou Y, Che Y, Huang Y. Knockdown of long non-coding RNA NEAT1 inhibits glioma cell migration and invasion via modulation of SOX2 targeted by miR-132. Mol Cancer. 2018;17:105.

44. He L, Qu L, Wei L, Chen Y, Suo J. Reduction of miR-132-3p contributes to gastric cancer proliferation by targeting MUC13. Mol Med Rep. 2017;15:3055-61.

45. Li G, Chong T, Yang J, Li H, Chen H. Kinesin Motor Protein KIFC1 Is a Target Protein of miR-338-3p and Is Associated With Poor Prognosis and Progression of Renal Cell Carcinoma. Oncol Res. 2018;27:125-37.

46. Wang B, Yu J, Sun Z, Luh F, Lin D, Shen Y, Wang T, Zhang Q, Liu X. Kinesin family member 11 is a potential therapeutic target and is suppressed by microRNA-30a in breast cancer. Mol Carcinog. 2020;59:908-22.

47. Sheng N, Xu YZ, Xi QH, Jiang HY, Wang CY, Zhang Y, Ye Q. Overexpression of KIF2A is Suppressed by miR-206 and Associated with Poor Prognosis in Ovarian Cancer. Cell Physiol Biochem. 2018;50:81022. 
48. Logan CY, Nusse R. The Wnt signaling pathway in development and disease. Annu Rev Cell Dev Biol. 2004;20:781-810.

49. Talbot LJ, Bhattacharya SD, Kuo PC. Epithelial-mesenchymal transition, the tumor microenvironment, and metastatic behavior of epithelial malignancies. Int J Biochem Mol Biol. 2012;3:117-36.

50. Guo H, Zhang X, Chen Q, Bao Y, Dong C, Wang X. miR-132 suppresses the migration and invasion of lung cancer cells by blocking USP9X-induced epithelial-mesenchymal transition. Am J Transl Res. 2018;10:224-34.

51. You J, Li Y, Fang N, Liu B, Zu L, Chang R, Li X, Zhou Q. MiR-132 suppresses the migration and invasion of lung cancer cells via targeting the EMT regulator ZEB2. PLoS One. 2014;9:e91827.

52. Zhao JL, Zhang L, Guo X, Wang JH, Zhou W, Liu M, Li X, Tang H. miR-212/132 downregulates SMAD2 expression to suppress the G1/S phase transition of the cell cycle and the epithelial to mesenchymal transition in cervical cancer cells. IUBMB Life. 2015;67:380-94.

53. Gu S, Liang H, Qi D, Mao L, Mao G, Qian L, Zhang S. Knockdown of KIF26B inhibits breast cancer cell proliferation, migration, and invasion. Onco Targets Ther. 2018;11:3195-203.

Figures
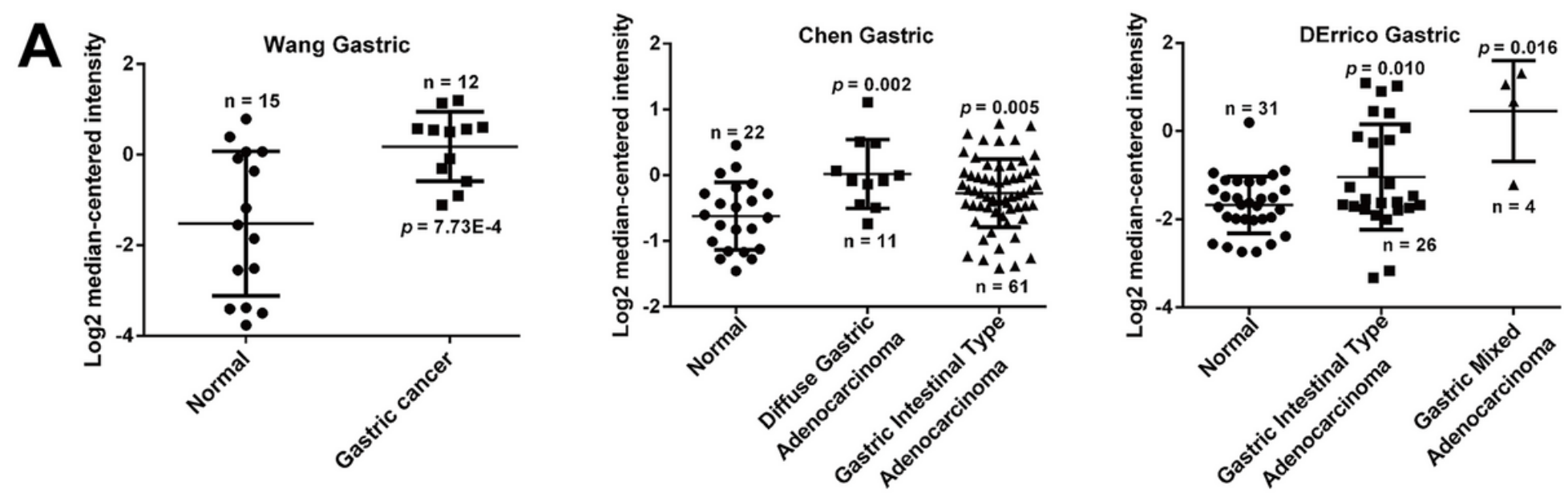

B
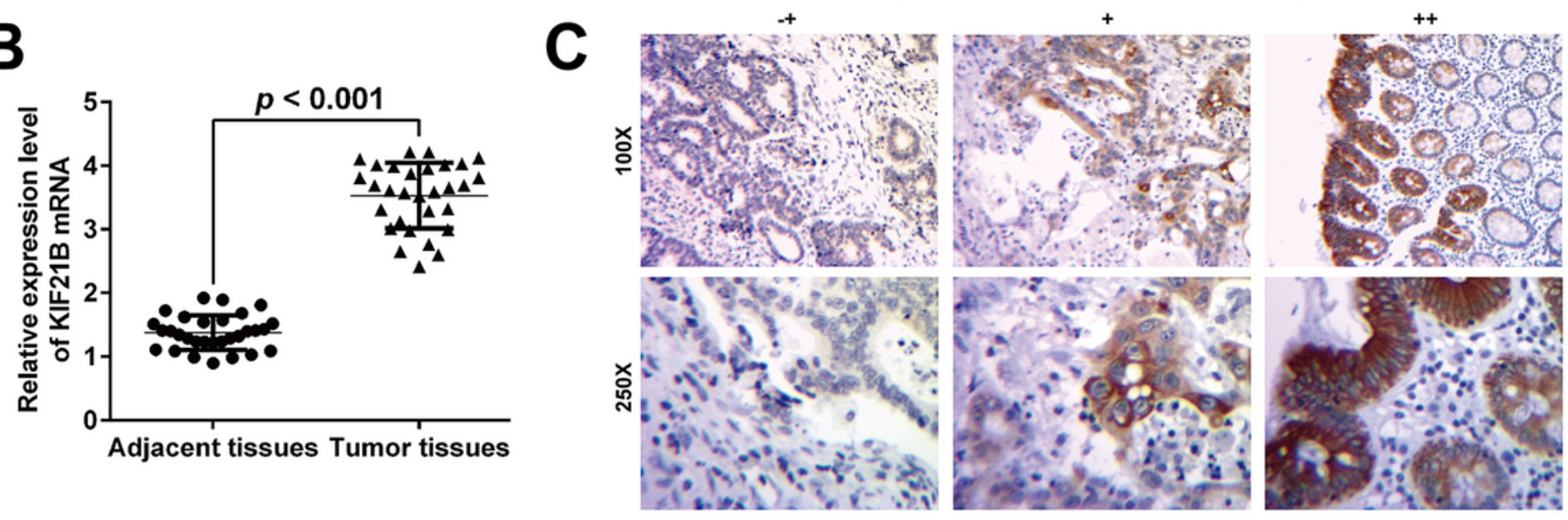

Figure 1 
Expression of KIF21B mRNA and protein levels in GC tissues; (A) The mRNA expression of KIF21B in Oncomine datasets including Wang Gastric, Chen Gastric and DErrico Gastric. (B) Expression of KIF21B mRNA was determined using reverse transcription quantitative PCR in 30 pairs of GC tissues and adjacent tissues. (C) Representative immunohistochemical staining of KIF21B in GC tissues and noncancerous tissues $(-+$, weak staining, + moderate staining, ++ strong staining).

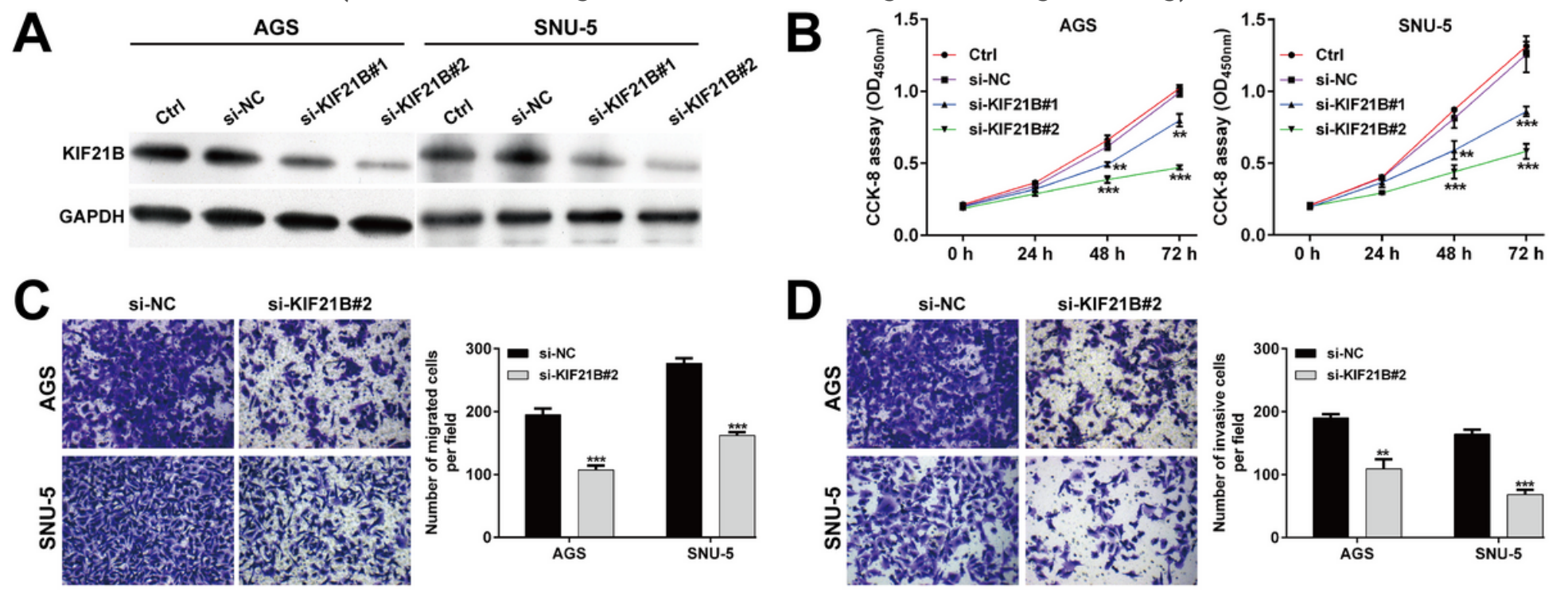

Figure 2

Effects of KIF21B knockdown on cell proliferation, migration and invasion in GC cells. (A) Western blot analysis was performed to detect KIF21B protein expression in AGS and SNU-5 cells after transfection with si-NC, si-KIF21B\#1 or si-KIF21B\#2 for 48 h. (B) CCK-8 assay was used to evaluate cell proliferation ability in AGS and SNU-5 cells. (C) Cell migration and (D) invasion in the analyzed groups (magnification, $\times 100$ ). All data are expressed as the means $\pm S D$. ${ }^{\star \star} p<0.01,{ }^{\star \star \star} p<0.001$, compared with si-NC group 
A

WT KIF21B 3'-UTR 5'...AGAAUCCGUCCGCAUGACUGUUU....3'

hsa-miR-132-3p 3' GCUGGUACCGACAUCUGACAAU 5'

MUT KIF21B 3'-UTR 5'...AGAAUCCGUCCGCAUAACCACCU...3'

B

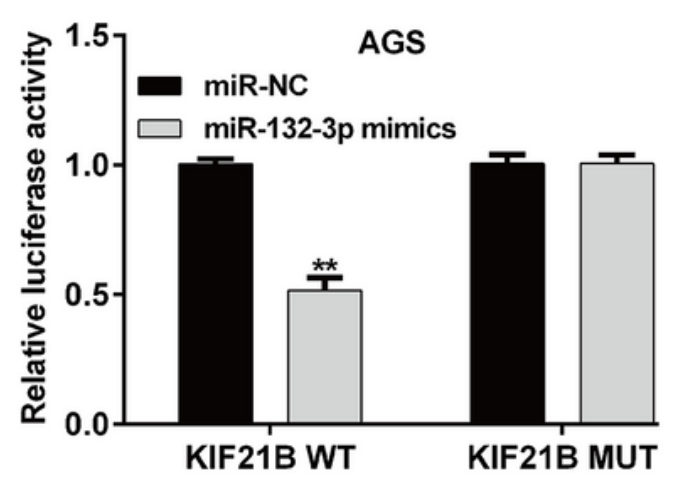

D

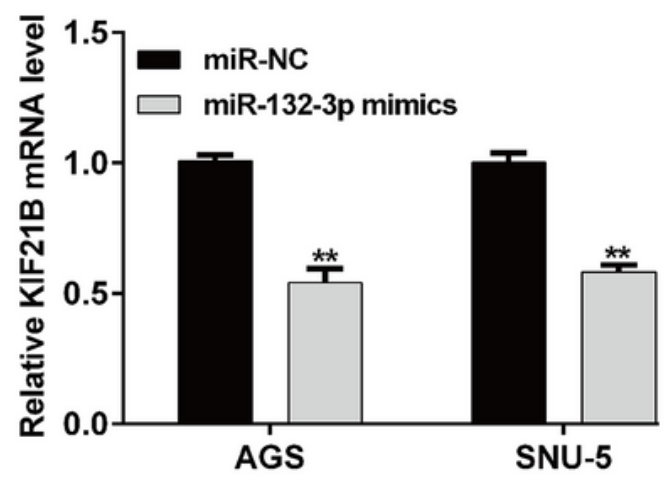

$\mathbf{F}$

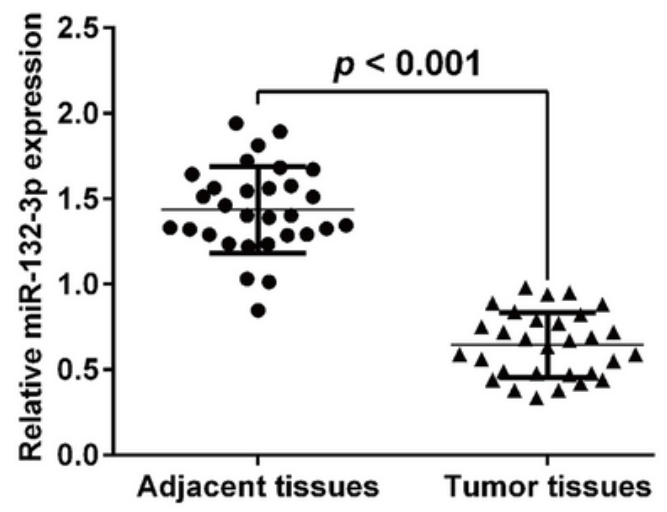

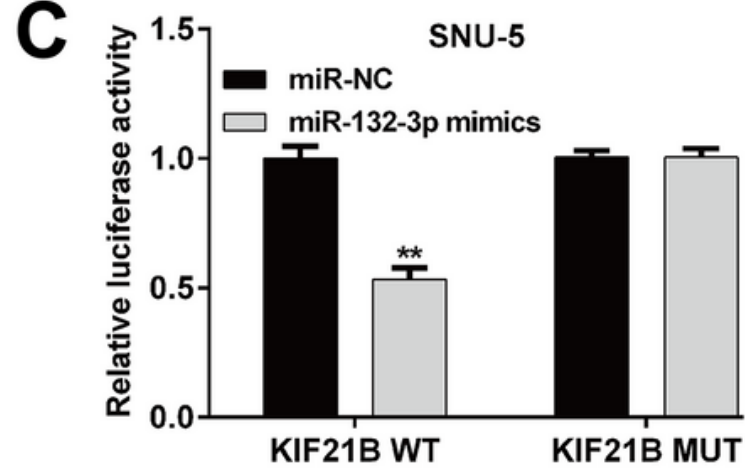

$\mathbf{E}$

miR-NC

miR-132-3p mimics

KIF21B

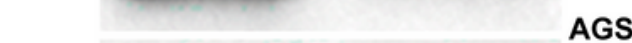

GAPDH

KIF21B
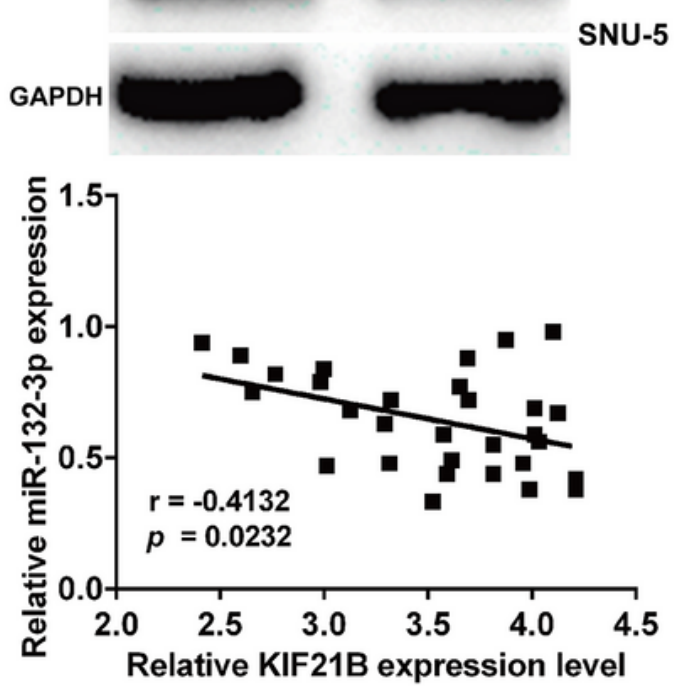

Figure 3

KIF21B was a target gene of miR-132-3p. (A) The potential binding sites of miR-132-3p and KIF21B mRNA, as well as the sequences in potential binding sites of mutant-type plasmid. Luciferase reporter assays were performed in (B) AGS and (C) SNU-5 cells with vectors including the putative miR-132-3p target sites in the 3'-UTR of KIF21B mRNA (wild type) and mutant-type. Data were normalized by Renilla/firefly luciferase activity. (D) Reverse transcription quantitative PCR and (E) western blot analysis 
was used to determine the expression levels of KIF21B in AGS and SNU-5 cells transfected with miR-132$3 p$ mimics or miR-NC. (F) Lower expression of miR-132-3p was observed in 30 pairs of GC tissues compared with matched adjacent tissues by reverse transcription quantitative PCR. (G) The correlation between KIF21B and miR-132-3p in 30 cases of GC clinical samples was analyzed by Spearman's rank correlation. All data are expressed as the means \pm SD. ${ }^{*} p<0.01$, compared with miR-NC group

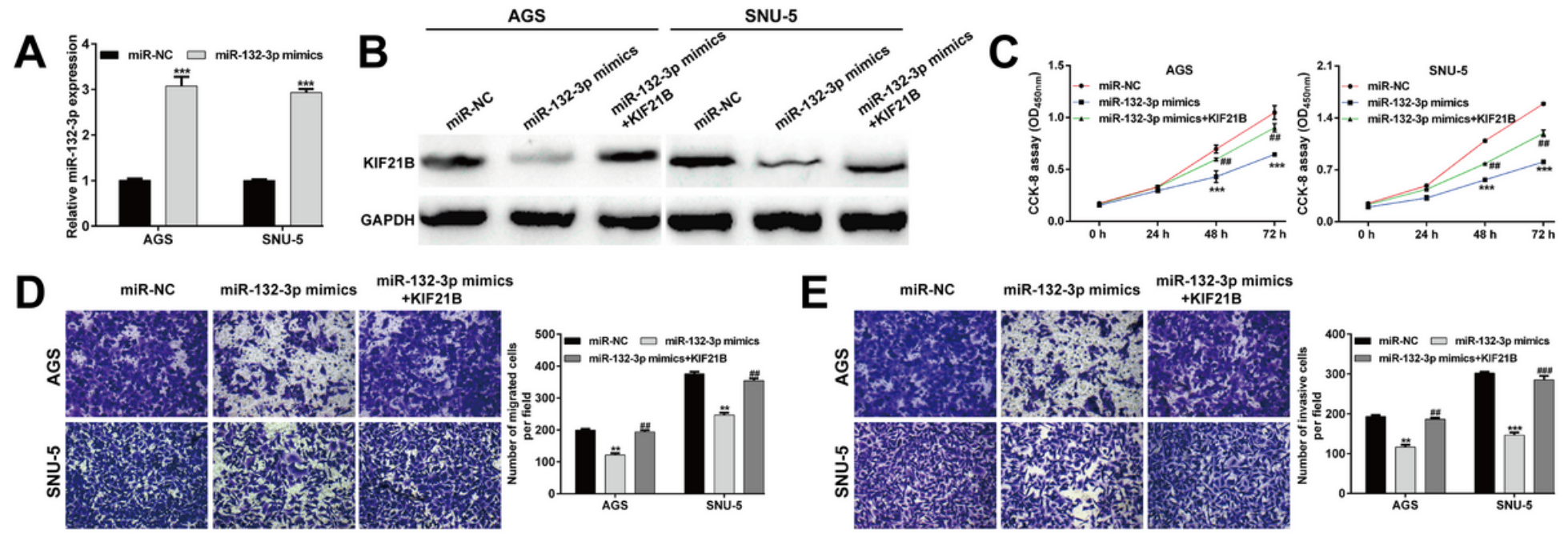

Figure 4

KIF21B alleviated the effects of miR-132-3p on cell proliferation, migration and invasion in GC cells. AGS and SNU-5 cells were transfected with miR-NC, miR-132-3p mimics or miR-132-3p mimics + KIF21B, respectively for $48 \mathrm{~h}$. (A) Reverse transcription quantitative PCR was used to determine miR-132-3p expression with U6 as internal control. (B) Western blot was used to determine the expression levels of KIF21B with GAPDH as a loading control. (C) CCK-8 assay was utilized to determine the cell proliferation in different groups. (D) Cell migration and (E) invasion ability was assessed by transwell assay in the above transfected cells. All data are expressed as the means \pm SD. ${ }^{*} p<0.01,{ }^{* *} p<0.001$, compared with miR-NC group; \#\#p < 0.01, \#\#\#p < 0.001, compared with miR-132-3p mimics group 


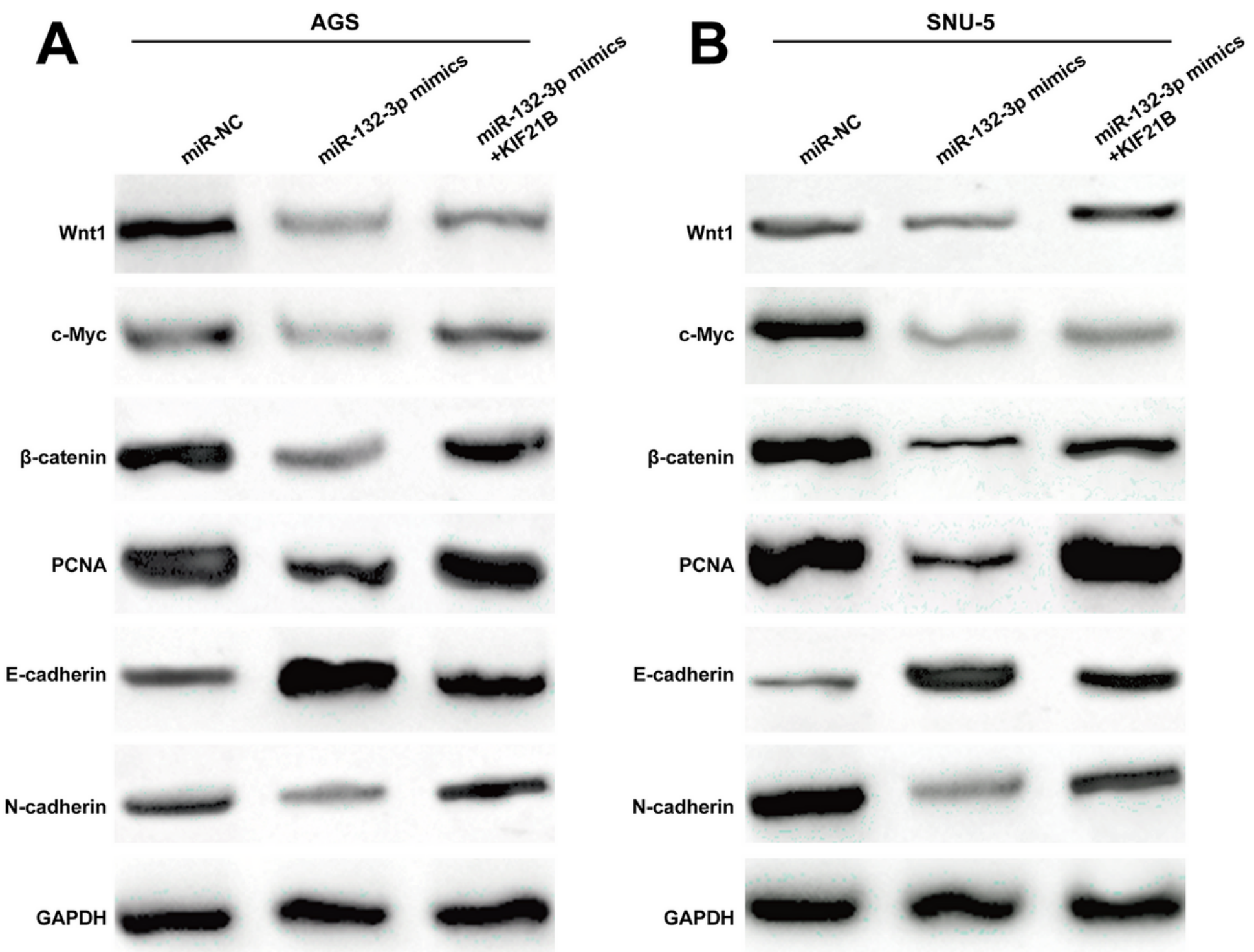

Figure 5

MiR-132-3p negatively regulated the Wnt/ $\beta$-catenin pathway by directly targeting KIF21B. AGS and SNU-5 cells were transfected with miR-NC, miR-132-3p mimics or miR-132-3p mimics plus KIF21B, respectively. Western blot assay was used to compare the protein levels of Wnt1, c-Myc, $\beta$-catenin, PCNA, E-cadherin and N-cadherin in transfected AGS (A) and SNU-5 (B) cells. GAPDH was used as an internal control. 